\title{
Influence of frequency resolution in case of frequency response function measurement in structural dynamics
}

\author{
Z. Gazdagh ${ }^{1, *}$, B. Vehovszky ${ }^{2}$ \\ ${ }^{1}$ Audi Hungaria ZRt, Whole Vehicle Engineering, \\ Győr, Audi Hungária út 1. H-9027, Hungary \\ ${ }^{2}$ Széchenyi István University, Department of Whole Vehicle Engineering, \\ Györ, Egyetem tér 1. H-9026, Hungary \\ *e-mail: zoltan.gazdagh@audi.hu
}

Submitted: 10/05/2021; Accepted: 18/08/2021; Published online: 16/09/2021

Abstract: Frequency resolution is an essential parameter in acoustical testing, even if we are using numerical or experimental method, for example when determining frequency response function (FRF) of a dynamic mechanical system, or executing modal analysis based on the FRFs. Finer resolution leads to more accurate results, at the expense of longer calculation/measurement process and larger data size. This parameter is generally set based on rules of thumb, prior practice or with big margin for safety. This results in waste time and data storage if the required frequency resolution is overestimated, or even significant errors in the results, if it is underestimated. Present paper offers a direct, method for the conscious determination of optimal frequency resolution. It is based fully on theoretical considerations, and investigates amplitude and phase distortion at resonances as target parameters. Beside defining the steps of the process, it is tested on a real structure, and the results are presented as well, proving the applicability and the appropriateness of the method. With this method, development engineers get a practical tool for adjusting the parameters of dynamic measurements and simulations.

Keywords: transfer function; frequency resolution; dynamic analysis; engineering acoustics 


\section{Introduction}

During prototype testing, dynamic measurements are vital, some of them are mandatory to validate virtual results in concept phase. The low frequency measurements are often referred to vehicle comfort, the mid frequency measurements are used for validating the global vehicle dynamics and used in acoustic assessments [1]. The transfer function (or frequency response function, FRF) measurement is one of the most frequently used method in dynamic assessment of vehicle parts or structures. The typical input parameters, that help the engineers to understand local and global dynamics, are transfer functions, auto- and crosspower functions as well as the coherence curves. These parameters are dependent on each other as well. They are the input for trimmed body experimental modal analysis, but they can be input parameters for the evaluation of equivalent torsional stiffness of the chassis as well. In general, transient or stationary excitation types are used for frequency response function measurements in automotive industry. Measurement parameter settings mostly depend on prior results and experience, however inappropriate frequency resolution may lead to imprecise damping calculation and magnitude distortion, when these values are determined from the FRFs with the widely used half-power bandwidth method [2]. The current study focuses on the understanding and quantification of these effects.

Several parameters, such as structural damping, frequency resolution, or the frequency range of the modal base, play an important role in the accuracy of excited vibration analysis. In case of simulation, the frequency response functions (FRFs) of the investigated structure are often synthetized. This simulation based FRF synthesis is highly dependent on the used modal range or usage of lower and upper compensation of modes - when residual compensation is not used, the upper frequency limit of the modal range should be at least twice of the upper limit of the frequency range used for the FRF synthesis. Another dominant parameter is the frequency resolution. It must be chosen sufficiently small to minimize the amplitude distortion of the response functions, but this parameter determines the length of the FFT blocks during data processing as well. The better the frequency resolution, the longer the acquisition time $(1 \mathrm{~Hz}$ resolution requires 1 second of data, but $0.1 \mathrm{~Hz}$ needs 10 seconds during a single acquisition without averaging), moreover, the larger the recorded data.

Equation (1) can be found in the user guide of MSc Nastran finite element solver [2]. The equation is applied for a single resonance peak and splits the half power bandwidth to $\mathrm{n}$ discrete points. This equation provides guidance for a possible determination of the correct frequency resolution in case of $\xi<0.1$. 


$$
\Delta \omega=\frac{2 \cdot \xi \cdot \omega_{r}}{n-1}
$$

In the context of (1), $\xi$ denotes the damping ratio, $\omega_{r}$ denotes the resonant frequency, $n$ is the number of discrete points in half power bandwidth and $\Delta \omega$ is the frequency resolution. However, when using this equation, the number of points (n) is up to the user, and the method does not take into account the level of amplitude distortion and frequency shift. In the following part, a newly developed, supplementary method is presented that takes into consideration the above effects as well, which are influenced by acquisition parameter settings.

\section{Considerations on amplitude distortion}

Let's focus on a single mass-spring-damper system, where the frequency transfer function can be written in the following form [3]:

$$
H_{d}(j \omega)=\frac{\frac{1}{m \omega^{2}}}{\left[\left(\frac{\omega_{r}}{\omega}\right)^{2}-1\right]+2 j \xi \cdot\left(\frac{\omega_{r}}{\omega}\right)}
$$

This relationship describes the receptance $\left(\mathrm{H}_{\mathrm{d}}\right.$, i.e. the displacement response divided by the single exciting input) when system is excited by a unit force at a frequency of $\omega$, its mass is $m$ and damping is $\xi$.

In acoustic measurements, however, the velocity response function (the so-called mobility equation) of the structure is generally required, which can be obtained by multiplying the displacement response function (2) by $j \omega=\frac{\omega}{-j}$ (the relation $j=-\frac{1}{j}$ can easily be understood as $\frac{j}{j}=1$ so $\frac{1}{j}=\frac{1}{j} \cdot \frac{j}{j}=\frac{j}{-1}=-j$ ). Aiming further simplification, by multiplying the nominator and the denominator equally by $\left(\frac{\omega}{\omega_{r}}\right)$, the resulted transfer function (velocity response or mobility equation) can be written as:

$$
H_{v}(j \omega)=\frac{\frac{1}{m \omega_{r}}}{j\left(\frac{\omega}{\omega_{r}}\right)-j\left(\frac{\omega_{r}}{\omega}\right)+2 \xi}
$$

In order to quantify the amount of distortion caused by the chosen frequency resolution, lets perturb the transfer function equation (3) with $\pm \frac{\Delta \omega}{2}$ frequency shift (referring to the $\Delta \omega$ frequency resolution), which represents the distance between 
the theoretical and the actually acquired frequency value. As a result, the new transfer function can be written as:

$$
H_{v}\left(j\left(\omega \pm \frac{\Delta \omega}{2}\right)\right)=\frac{\frac{1}{m \omega_{r}}}{j\left(\frac{\omega \pm \frac{\Delta \omega}{2}}{\omega_{r}}\right)-j\left(\frac{\omega_{r}}{\omega \pm \frac{\Delta \omega}{2}}\right)+2 \xi}
$$

We are looking for the worst case, so when the actual frequency $(\omega)$ is located in the middle of two acquired, neighbouring frequency values $\left(\omega-\frac{\Delta \omega}{2}\right.$ and $\omega+\frac{\Delta \omega}{2}$, see Fig. 1 for the case when $\left.\omega=\omega_{r}\right)$. The amplitude distortion $\varepsilon_{H}(j \omega)$ for such case can be given by dividing the acquired amplitude (4) by the theoretical one (3), resulting:

$$
\varepsilon_{H}(j \omega)=\left|\frac{H_{v}\left(j\left(\omega \pm \frac{\Delta \omega}{2}\right)\right)}{H_{v}(j \omega)}\right|=\left|\frac{j\left(\frac{\omega}{\omega_{r}}\right)-j\left(\frac{\omega_{r}}{\omega}\right)+2 \xi}{j\left(\frac{\omega \pm \frac{\Delta \omega}{2}}{\omega_{r}}\right)-j\left(\frac{\omega_{r}}{\omega \pm \frac{\Delta \omega}{2}}\right)+2 \xi}\right|
$$

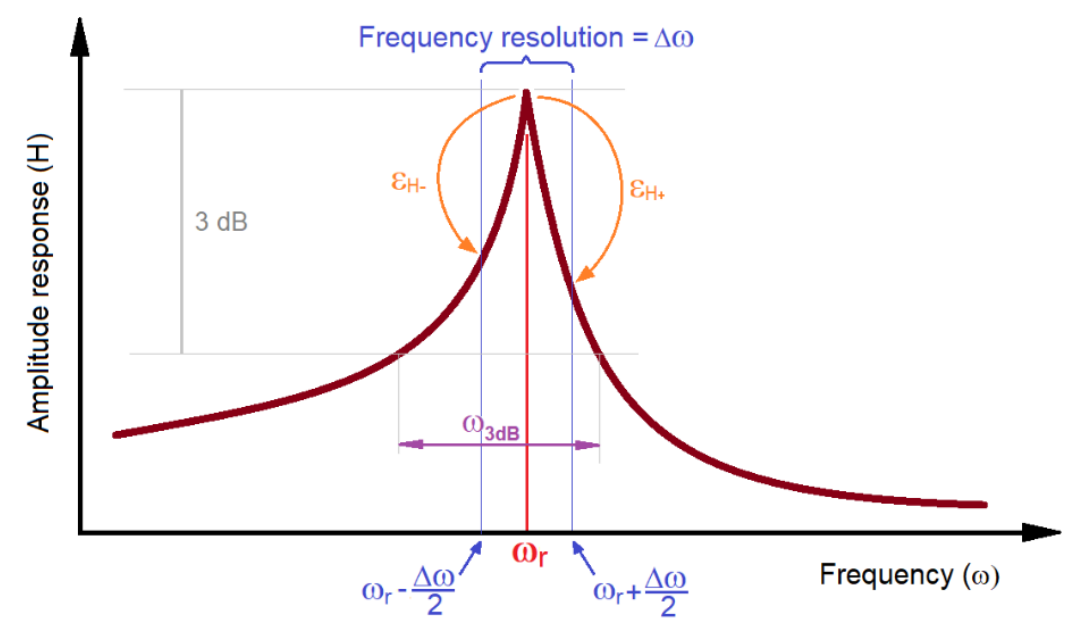

Figure 1. Resonance peak and frequency resolution - worst case 
Consider the amplitude distortion at resonance frequency $\omega_{r}$ in the following. Substituting $\omega=\omega_{r}$ in equation (5) and highlighting $\frac{ \pm \Delta \omega}{2 \omega_{r}}$, after performing the simplifications, the obtained amplitude distortion is:

$$
\varepsilon_{H}\left(j \omega \mid \omega=\omega_{r}\right)=\frac{1}{\left|j\left(\frac{1}{2 \xi} \cdot \frac{ \pm \Delta \omega}{2 \omega_{r}} \cdot \frac{2+\frac{ \pm \Delta \omega}{2 \omega_{r}}}{1+\frac{ \pm \Delta \omega}{2 \omega_{r}}}\right)+1\right|}
$$

Being a complex function, as a result of the absolute value calculation, the following equation is obtained:

$$
\varepsilon_{H}\left(j \omega \mid \omega=\omega_{r}\right)=\frac{1}{\sqrt{\left(\frac{1}{2 \xi} \cdot \frac{ \pm \Delta \omega}{2 \omega_{r}} \cdot \frac{2+\frac{ \pm \Delta \omega}{2 \omega_{r}}}{1+\frac{ \pm \Delta \omega}{2 \omega_{r}}}\right)^{2}+1}}
$$

Using equation (7) the level of maximal amplitude distortion $\varepsilon_{H}$ can be determined at resonance, which is the function of $\xi$ (damping ratio), $\omega_{r}$ (resonance frequency) and $\pm \frac{\Delta \omega}{2}$ frequency resolution. Note: this value is the theoretical maximum distortion, when the resonance frequency $\left(\omega_{r}\right)$ falls exactly in the middle of two neighbouring, acquired frequency values (see Fig. 1.) The best case is when the acquired frequency hits exactly the resonance frequency, in this case there is no distortion, $\varepsilon_{H}=1$.

In order to represent the practical applicability of equation (7), the $\varepsilon_{H}$ amplitude distortion is plotted as a function of $\frac{\Delta \omega}{2 \omega_{r}}$ in Fig. 2. Curves are plotted for different damping ratios to visualize the influence of damping. Lower damping shows higher risk of distortion.

It is worth mentioning that the amplitude distortion in plus and minus direction is not equal as resonance curves are not symmetric (Fig. 1.), however, the difference between $\varepsilon_{H_{+}}$and $\varepsilon_{H_{-}}$is negligibly small, thus Fig. 2. illustrates the average of the two values. 


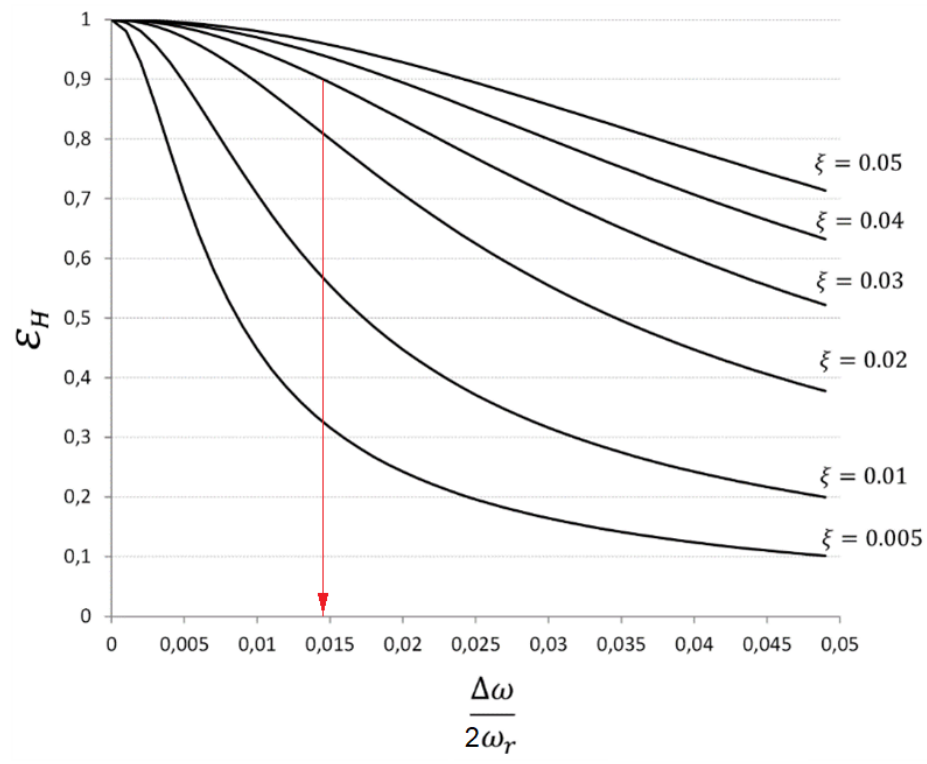

Figure 2. $\varepsilon_{H}$ amplitude distortion as a function of $\frac{\Delta \omega}{2 \omega_{r}}$

\section{Optimal parameters for FRF measurements}

Let us consider the practical application of the above findings: Using the curves of Fig. 2 and setting the permissible distortion to be $90 \%$ (meaning $\varepsilon_{H} \geq 0.9$ ) and estimating the damping ratio to be $3 \%(\xi=0.03)$, the maximum allowable $\frac{\Delta \omega}{2 \omega_{\mathbf{r}}}$ value is 0.014 , meaning $\Delta \omega=2.8 \mathrm{~Hz}$ maximum allowable frequency resolution beside $\omega_{\mathrm{r}}=100 \mathrm{~Hz}$ resonance frequency. By decreasing $\omega_{\mathrm{r}}$ to $50 \mathrm{~Hz}$, maximum allowable frequency resolution decreases to $1.4 \mathrm{~Hz}$. In short: the method gives us direct values for frequency resolution, assuming a maximum damping ratio and giving the lowest frequency limit of interest.

In the following, let's examine the amplitude distortion $\varepsilon_{H}$ in relation with the half-power bandwidth range. For this, first take a look at the correlation of damping ratio and half-power bandwidth. As it is known, the damping ratio at resonance can be calculated by the following equation [4]:

$$
\xi=\frac{\omega_{2}-\omega_{1}}{\omega_{r}\left(\tan \frac{\theta_{1}}{2}+\tan \frac{\theta_{2}}{2}\right)}
$$


where $\theta_{1}$ and $\theta_{2}$ are the angles between $\omega_{r}$ and $\omega_{1}$, and $\omega_{r}$ and $\omega_{2}$, respectively; $\omega_{1}$ and $\omega_{2}$ being the lower and upper frequency limits or the half power $(3 \mathrm{~dB})$ frequency band. Assuming $\xi \leq 0.1$, the relative phase angles at both ends of the $3 \mathrm{~dB}$ bandwidth $\left(\theta_{1}\right.$ and $\left.\theta_{2}\right)$ are close to $90^{\circ}$, so the equation can be simplified to the following form [5]:

$$
\xi=\frac{\omega_{3 d B}}{2 \omega_{r}}
$$

$\omega_{3 d B}$ being the half-power bandwidth $\left(\omega_{2}-\omega_{1}\right)$.

As a general rule, in case of $\xi$ above 0.1 , the calculation of $3 \mathrm{~dB}$ bandwidth using equation (9) is inaccurate, and only equation (8) can be used for damping estimation, while below 0.1 equation (9) gives adequate accuracy, meaning that the half-power bandwidth is assumed to be proportional to the damping ratio.

For further consideration, substitute the rearranged format of formula $\left(\frac{\Delta \omega}{2 \cdot \xi \cdot \omega_{r}}=\frac{1}{n-1}\right)$ in equation (7). The result is as follows:

$$
\varepsilon_{H}\left(j \omega \mid \omega=\omega_{r}\right)=\frac{1}{\sqrt{\left( \pm \frac{1}{2(n-1)} \cdot \frac{2 \pm \frac{\xi}{n-1}}{1 \pm \frac{\xi}{n-1}}\right)^{2}+1}}
$$

Equation (10) determines the amplitude distortion $\varepsilon_{H}$ as a function of $\mathrm{n}$ (the number of discrete frequency values in the half-power bandwidth). Fig. 3. illustrates it graphically, by giving the amplitude distortion for $\omega_{r}-\frac{\Delta \omega}{2}$ and $\omega_{r}+\frac{\Delta \omega}{2}$ for $\xi=0.1$ case (grey) and the average of the two (black). The difference of the two curves tends firmly to zero when damping ratio is decreased. The inset diagram shows the average curves only for different damping values. One can conclude that the amplitude distortion does not depend directly on the resonance frequency (see equation (10)), moreover, the dependence on damping is negligible too when $\xi \leq 0.1$.

Note: Fig. 2 and 3 both demonstrates the amplitude distortion, but the former focuses on the frequency resolution (which is important from practical point of view), while the latter examines the number of discrete frequency points within the half-power bandwidth, which is more useful for analytical considerations. The correlation of the two is given by equation (1). 


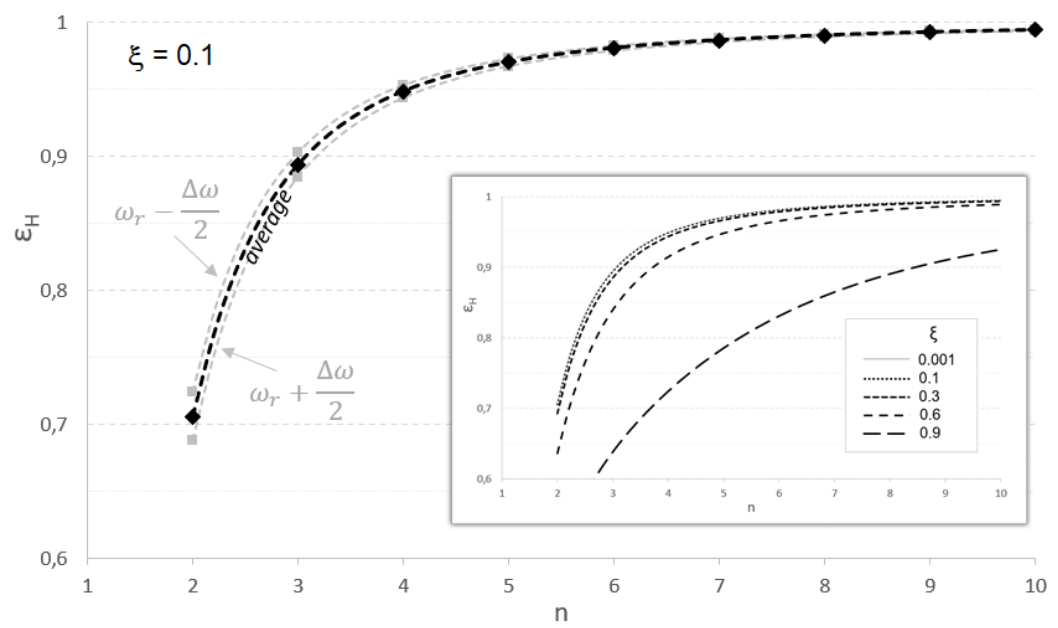

Figure 3. $\varepsilon_{H}$ amplitude distortion as a function of number of discrete points in the half power bandwidth of a resonance at a constant damping ratio $(\xi=0.1$ for the base diagram and for different $\xi$ values for the inset)

By using the detailed correlations, the maximum error of amplitude distortion due to frequency discretization can be simply controlled, which gives us useful suggestions when setting acquisition parameters during dynamic measurements. This method can be used in simulations as well to reduce measurement-based error in hybrid simulation models. Such models are mentioned in [6] and [9]. Furthermore - as damping is generally calculated using the half-power bandwidth principle (equation (8) and (9)) into which the amplitude distortion also introduces error - the accuracy of damping calculation can also be kept under control.

The next examples show some further consequences of the appropriateness of frequency resolution value. Fig. 4. shows measured FRF curves in Nyquist diagram, from which the importance of frequency resolution is obvious: Even though measuring with coarser resolution is faster, results may be practically unusable.

One good method to check the adequacy of frequency resolution is plotting the FRF curves in Nyquist diagram [7]. In order to validate the number of discrete points in half power bandwidth, an experimental test was carried out and the FRFs were plotted in Nyquist diagrams: Fig 4 shows the coarser, while Fig. 5 shows finer frequency resolution cases. 
Z. Gazdagh and B. Vehovszky - Acta Technica Jaurinensis, Vol. 14, No. 4, pp. 508-520, 2021
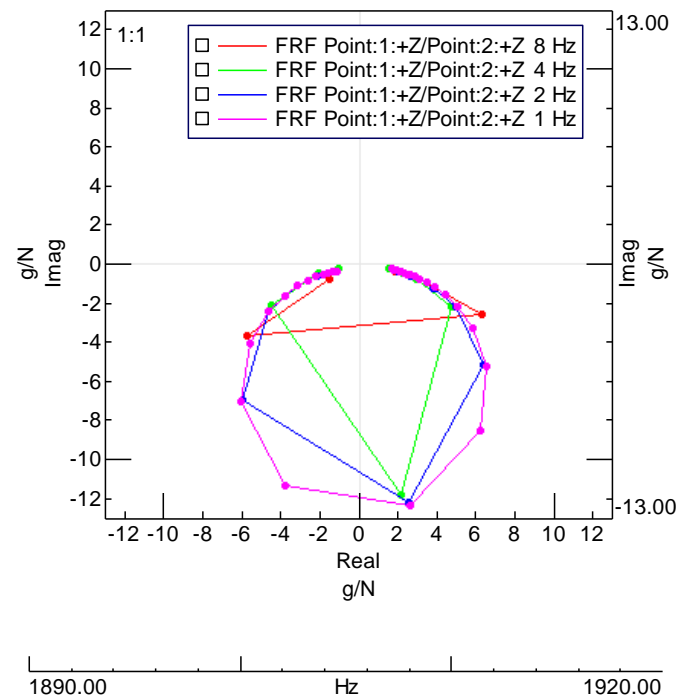

Figure 4. Measured FRFs with coarse frequency resolution
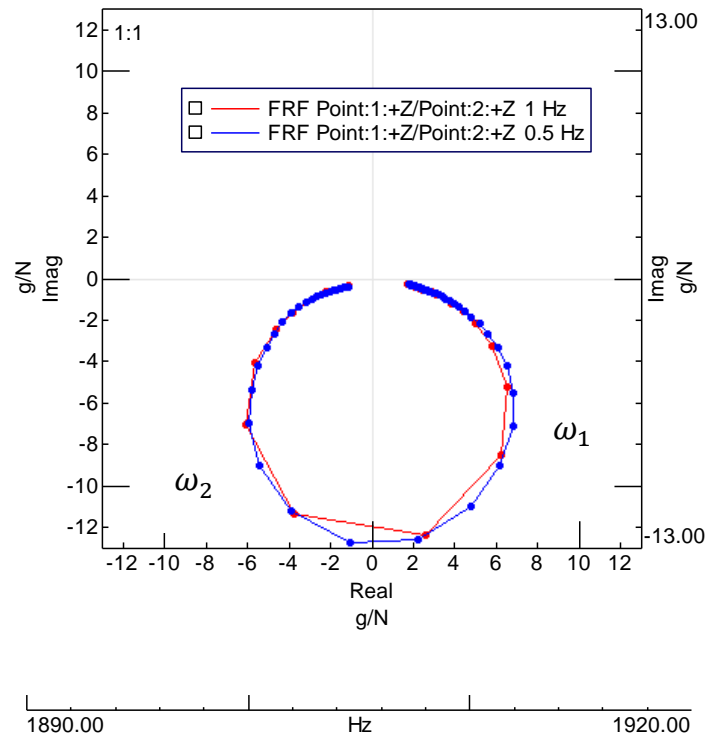

Figure 5. Nyquist diagram of FRFs with higher resolution around resonance 
The applied measurement method was the so-called impact testing $[8,12]$, with an average of 5 impact measurements. During this transient type testing, exponential window was set to be $100 \%$, but on force window $10 \%$ exponent was applied to remove possible noise from force signal after impact. For FRF estimation, the H1 estimation method was set, which is the most commonly used estimator [11], assuming all the noise to be on the output. The impact hammer was a low weight, modally tuned hammer with a steel tip, to ensure the high frequency energy input. The impact duration was around 0.3 milliseconds.

In Fig. 5 two cases with finer frequency resolution are compared for the same experiment (red curve with $1 \mathrm{~Hz}$ and blue curve with $0.5 \mathrm{~Hz}$ ). The two ends of the $3 \mathrm{~dB}$ bandwidth are marked with $\omega_{1}$ and $\omega_{2}$. In case of the red curve $\mathrm{n}=5$ and blue FRF gives $\mathrm{n}=9$. Amplitude distortions were calculated as $\varepsilon_{H, 1 \mathrm{~Hz}}=0.92$ and $\varepsilon_{H, 0.5 \mathrm{~Hz}}=0.976$.

The damping ratio and resonance amplitude were also calculated (Table 1.) One can see that coarser resolution result not only in larger amplitude distortion but inaccurate damping value as well.

Table 1. Damping ratio and FRF amplitude of resonance frequency

\begin{tabular}{|c|c|c|}
\hline $\begin{array}{c}\text { Frequency } \\
\text { Resolution }\end{array}$ & $\begin{array}{c}\text { Damping } \\
\text { ratio }\end{array}$ & $\begin{array}{c}\text { FRF } \\
\text { amplitude }\end{array}$ \\
\hline $1 \mathrm{~Hz}$ & $0.09 \%$ & $12.63 \mathrm{~g} / \mathrm{N}$ \\
\hline $0.5 \mathrm{~Hz}$ & $0.1 \%$ & $12.76 \mathrm{~g} / \mathrm{N}$ \\
\hline
\end{tabular}

The same FRFs are plotted in Bode diagram, see Fig 6. Small change in frequency resolution between the two FRF measurement lead already to a frequency shift of the resonance peak and produces different damping ratio when using the $3 \mathrm{~dB}$ bandwidth equation (8). 


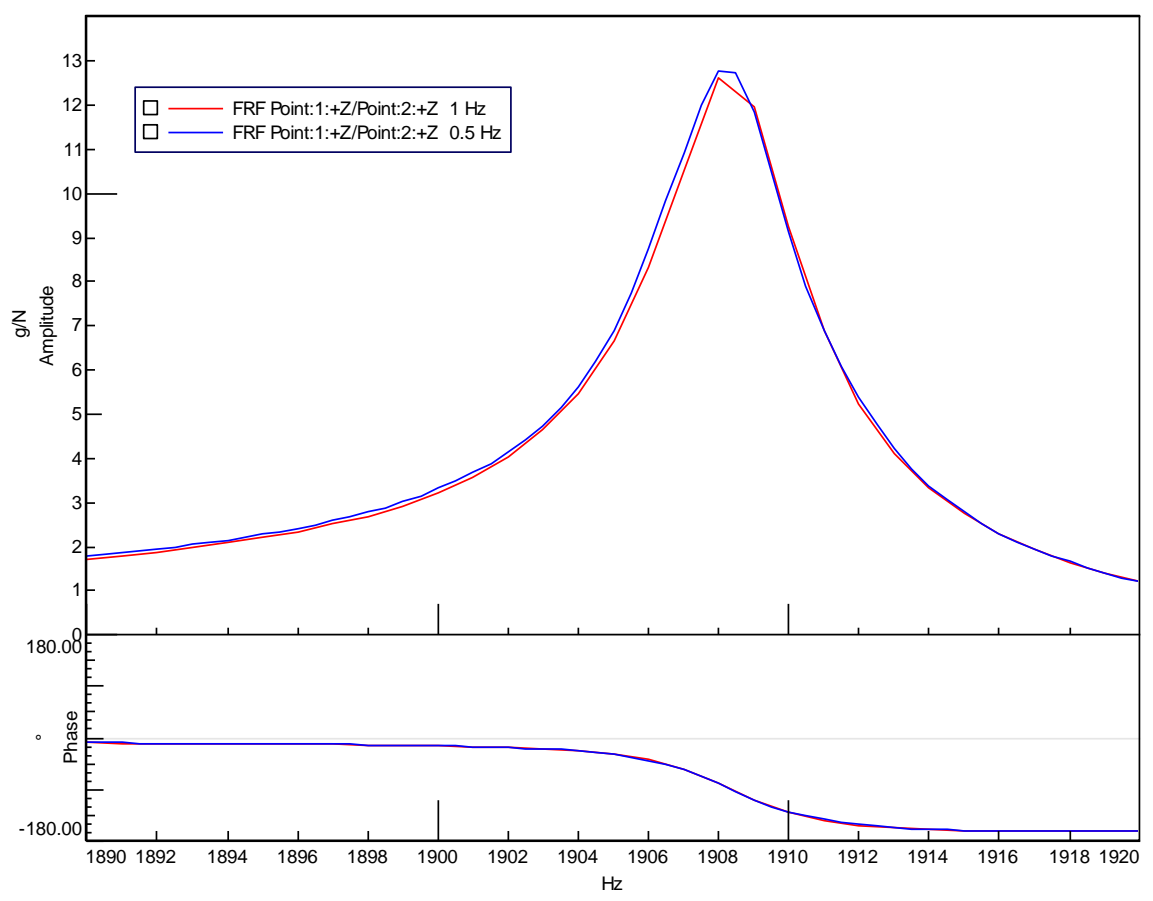

Figure 6. Bode Plot of FRFs near resonance

\section{Conclusion}

The method presented in this article offers a solution for the correct choice of the frequency resolution as a measurement setup parameter, taking into account the potential amplitude distortion of the FRFs. The level of distortion is determined as a function of frequency resolution, damping ratio and resonance frequency. Using this equation, one can determine the minimum frequency resolution which is required to keep the maximal amplitude distortion in a given range.

The number of discrete points within the half-power bandwidth is also evaluated, which makes easy to work with, as resonance frequency in this case is not a variable, and neither damping has significant influence (if lower than 0.1). By defining the appropriate criteria for measurement accuracy, the described functions and graphs provide good guidance for the selection of suitable parameters, so that amplitude of the frequency response function can be measured with pre-defined accuracy, and the error of damping value can be controlled as well. The application area covers the field of modal analysis [10] and other FRF based measurements. 
A potential continuation of the work is the analysis of the effect of exponential windowing used during the impact testing, which also causes pseudo-damping effect [13]. It would be useful to form similar direct suggestions for the optimal parameter selection.

\section{References}

[1] M. F. Treszkai, D. Feszty, Review of Hybrid Finite Element - Statistical Energy Analysis methods in vehicle NVH predictions, Acta Technica Jaurinensis 12 (4) (2019) pp. 347-370.

doi: https://doi.org/10.14513/actatechjaur.v12.n4.513

[2] MD Nastran Dynamic Analysis User's Guide, 2010.

[3] M. R. Hatch, Vibration simulation using MATLAB and ANSYS, Chapman \& Hall/CRC, Washington D.C., 2000.

[4] W. Heylen, S. Lammens, P. Sas, Modal Analysis Theory and Testing, $K U$ Leuven Academic Press, Belgium, 1999.

[5] M. Geradin, D. Rixen, Mechanical vibrations: theory and application to structural dynamics, Wiley, New York, 2015.

[6] P. Peeters, S. Manzato et al., Reducing the impact of measurement errors in FRF-based substructure decoupling using modal model, KU Leuven Academic Press, Belgium, 2018.

[7] M. F. Treszkai, D. Sipos, D. Feszty, Damping Determination by Half-Power Bandwidth Method for a Slightly Damped Rectangular Steel Plate in the MidFrequency Range, Acta Technica Jaurinensis 13 (3) (2020) pp. 177-196. doi: https://doi.org/10.14513/actatechjaur.v13.n3.545

[8] The Fundamentals of Modal Testing - Application Note 243-3, Agilent Technologies, 2000.

URL

https: //Www.modalshop.com/techlibrary/Fundamentals $\% 200 f \% 2$ omodal $\div 20$ Testing.pdf

[9] Z. Gazdagh, Z. Jamil, J. M. Allport, A model answer: hybrid vibration analysis, Cummins Turbo Technologies HTi Magazine 13 (2009) pp. 3-4. 
URL https://pure.hud.ac.uk/en/clippings/a-model-answerhybrid-vibration-analysis

[10] A. Schweighardt, B. Vehovszky et al., NVH and Modal Analysis in Vehicle Industry, Perner's Contacts Special Issue 2 (2019) pp. 258-266.

[11] Z. Mao, M. Todd, Statistical modeling of frequency response function estimation for uncertainty quantification, Mechanical Systems and Signal Processing, 38 (2) (2013) pp. 333-345.

doi: https://doi.org/10.1016/j.ymssp.2013.01.021

[12] D. L. Brown, R. J. Allemang, A. W. Phillips, Forty Years of Use and Abuse of Impact Testing: A Practical Guide to Making Good FRF Measurements, Experimental Techniques, Rotating Machinery and Acoustics 8 (2015) pp. 221-241.

doi: https://doi.org/10.1007/978-3-319-15236-3_21

[13] D. Tajiri, M. Matsubara, S. Kawamura, Identification of modal parameters in a lightly damped system based on impact vibration testing: Application of exponential window and removal of its effect, IOP Conf. Series: Journal of Physics 1264 (2019) 012011.

doi: https://doi.org/10.1088/1742-6596/1264/1/012011 